\title{
Guiding the Evolution of a Multilingual Ontology in a Concrete Setting
}

\author{
Mauro Dragoni $^{1}$, Chiara Di Francescomarino ${ }^{1}$, Chiara Ghidini ${ }^{1}$, Julia Clemente ${ }^{2}$, \\ and Salvador Sánchez Alonso ${ }^{2}$ \\ 1 FBK-IRST, Trento, Italy \\ 2 Universidad de Alcalá, Alcalá de Henares, Spain \\ \{dragoni, dfmchiara,ghidini\}afbk.eu, julia@aut.uah.es, \\ salvador.sanchez@uah.es
}

\begin{abstract}
Evolving complex artifacts as multilingual ontologies is a difficult activity demanding for the involvement of different roles and for guidelines to drive and coordinate them. We present the methodology and the underlying tool that have been used in the context of the Organic.Lingua project for the collaborative evolution of the multilingual Organic Agriculture ontology. Findings gathered from a quantitative and a qualitative evaluation of the experience are reported, revealing the usefulness of the methodology used in synergy with the tool.
\end{abstract}

\section{Introduction}

Ontologies are dynamic entities that evolve over time because they need to reflect changes in the domain they describe, in their conceptualization, or in their specification. As stated in [1], ontology evolution can be defined as "the timely adaptation of an ontology to the arisen changes and the consistent propagation of these changes to dependent artifacts.".

Managing the evolution of ontologies is a complex problem, well known to the Semantic Web community, and a number of efforts are devoted to tackle different aspects of the problem, as described in [2]. The problem becomes even more complex when, besides the evolution of the ontology entities, the changes are related also to the multilingual aspects of the ontology itself. Indeed, in the last years, the construction of multilingual ontologies has become an important objective for organizations working in multilingual environments. Examples are companies which need to apply ontologies to information retrieval on a mass of resources written in different languages, or international bodies using ontologies as a way of describing terminological standards in a particular field (e.g., food, diseases, agriculture, and so on). As described in [3], obtaining multilingual ontologies is a complex activity which requires to tackle a number of problems spanning from the translation of labels and description associated to a given ontology entity to the adaptation of the ontology to a concrete language and cultural community.

These requirements lead to the necessity of guiding the evolution of an ontology according to its final use; moreover, this necessity become stronger when ontologies are used not only for modeling a domain, but also for describing and accessing resources in knowledge repositories or web portals. 
In this paper we present the methodological and technical solution used for facing the problem of the multilingual evolution of an ontology for the organic agriculture (the Organic Agriculture ontology) in the context of the Organic.Lingua project.

The main contributions of the paper are: (i) the definition of a methodology for guiding the evolution in a collaborative environment and its usage in a real setting; and (ii) a quantitative and a qualitative evaluation about the synergistic usage of a semantic web tool and the defined methodology.

\section{The Organic.Lingua Project}

Organic.Lingua (http://www.organic-lingua.eu) is an EU-funded project that aims at providing automated multilingual services and tools facilitating the discovery, retrieval, exploitation and extension of digital educational content related to Organic Agriculture and AgroEcology. More in concrete, the project aims at providing, on top of a web portal, cross-lingual facility services enabling users to (i) find resources in languages different from the ones in which the query has been formulated and/or the resource described (e.g., providing services for the cross-lingual retrieval); (ii) manage meta-data information for resources in different languages (e.g., offering automated meta-data translation services); and (iii) contribute to evolve the content (e.g., providing services supporting the users in the content generation).

The accomplishment of these objectives is reached in the Organic.Lingua project by means of two components: on the one hand, a web portal offering software components and linguistic resources able to provide multilingual services and, on the other hand, a conceptual model (formalized in the Organic Agriculture ontology) used for managing information associated with the resources provided to the final users and shared with other components deployed on the Organic.Lingua platform. In a nutshell, the usage of the Organic Agriculture ontology is twofold:

- Resource annotation: each time a content provider inserts a resource in the repository, the resource is annotated with one or more concepts extracted from the ontology. The list of available concepts is retrieved by using an ontology service deployed in the ontology management component (shown in Section 4). Then, this list is exploited for annotating the learning resources published on the Web portal.

- Resource retrieval: when web users perform queries on the system, the ontology is used, by the back-end information retrieval system, to perform advanced searches based on semantic techniques. Moreover, the ontology is used also by the CrossLanguage Information Retrieval component for query expansion purposes.

Due to this intensive use of the ontology in the entire Organic.Lingua portal, evolving both the knowledge represented by the artifact, as well as, the linguistic layer, requires a precise methodology, and dedicated tools, for avoiding the loss of effectiveness of the components deployed on the platform. In the next two sections we describe the methodology that we have defined in the context of the Organic.Lingua project and that we propose as a general best practice for the evolution of complex artifacts as the ones used in this project (Section 3) as well as the dedicated collaborative tool that supports the proposed methodology (Section 4). 


\section{Guiding Evolution with a Scenario-Based Methodology}

Collaboratively building, maintaining and evolving multilingual ontologies is not a trivial task: multilinguality, in fact, adds the linguistic problem to classical problems of collaborative modeling such as the background, skills and role differences that may exist between e.g., domain and knowledge experts that collaboratively model the ontology. Facing all these issues together requires appropriate methodologies (and tools) to support the work of the involved experts.

In the Organic.Lingua project, a multi-role scenario-based methodology (MRSB) was proposed and adopted for the Organic Agriculture ontology evolution. The methodology relies on involving experts playing different roles and guiding them, step-by-step through tasks and critical scenarios, towards the collaborative evolution of the multilingual ontology.

\subsection{MRSB: A Multi-Role Methodology}

Three different types of experts are involved in the ontology evolution process: domain experts, language experts and knowledge engineers. Domain experts play a key role as they are in charge of driving the core changes in the ontology; language experts have to revise existing translations and to provide new translations for the newly added terms; finally, knowledge engineers provide a general support for the evolution process and ensure correctness from a formal point of view.

To coordinate all these different experts, the MRSB methodology proposes to guide them step-by-step through tasks and guidelines. In detail:

- domain experts are asked to create or contribute to discussions, suggesting actions to be taken on the ontology and/or commenting on existing issues: they are recommended to clearly state whether all the suggestions should be implemented, if only some and why;

- once reached an agreement, knowledge engineers can be notified and take the final decision: either approve or discard the suggestion and proceed with the update on the English version of the ontology;

- finally, the language experts are asked to provide, check and revise the translations of labels and definitions, often produced by translation services.

\subsection{MRSB: A Scenario-Based Methodology}

The MRSB methodology proposes to guide domain experts in the ontology evolution through critical scenarios which represent the major types of changes that have arisen from the analysis carried out for the Organic Agriculture ontology. Such scenarios address three main categories of activities: the general revision of the ontology (from scenario 1 to 3 ), the revision of the ontology by taking into account environments in which ontologies are exploited by external tools (scenarios 4 and 5), and, finally, the revision of the linguistic layer. To further guide the experts in the evolution process, the scenarios have been complemented with the results of a rigorous analysis carried out by knowledge engineers on the use of the entities in the existing version of the ontology 
(e.g., which terms are less frequently used for searches, how many resources are retrieved by different terms). This choice is motivated by the fact that often ontologies are not used only for representing the knowledge of a particular domain, but they are also exploited for other tasks like the annotation and the retrieval of resources with/through semantic information.

The devised scenarios are the following:

1. Entity Deprecation Scenario. A complete analysis of the active version of an ontology, generally performed jointly by the domain experts and the knowledge engineers, may lead to a report containing a set of instances considered unnecessary for the representation of the target domain. Therefore, domain experts are asked to identify all candidate entities for removal and start the relative discussions, with the support of the knowledge engineers, for reaching an agreement about the status of each identified concept.

2. Ontology Mapping Scenario. A linguistic analysis of the terms used for defining entities in an ontology, may lead to the consideration that some of the terms used for defining the entities generate ambiguities when a user needs to understand the meaning of the label. This scenario is designed for finding a solution to this problem by asking domain experts to find concepts, in external domain-related knowledge bases (KBs), that may be linked with the ones defined in the ontology that they are revising. Moreover, it is also desirable that, the terminology used for revising the ontology, is the same used in the external knowledge base.

3. Ontology Enrichment Scenario. In this scenario, domain experts are asked to complete the ontology by including concepts in those areas that were more poorly covered. For accomplishing this task, domain experts are invited to consult domainrelated resources (concept lists, knowledge bases, etc.) for finding new concepts and relationship not previously described in the ontology.

4. Entity Specialization Scenario. This scenario addresses the necessity of increasing the granularity of some branches of the ontology. Such an increase is required, not only for the completeness of the ontology, but also when there are concepts used for annotating a huge number of resources, e.g., terms qualifying up to 500 resources or more. Indeed, a huge set of results is not useful in searches, because it does not really help users to find effective results.

5. Entity Generalization Scenario. The opposite of what has been described in the previous scenario happens when some concepts have been used for annotating a very small number of resources or are not used at all. In this case, domain experts are appointed to study each particular case in detail and to suggest, eventually, the deprecation for those concepts that lead to the retrieval of few resources. As for the previous scenario, the number of retrieved resources is used as a distinguishing parameter for evaluating which kind of action the domain experts should propose for evolving the ontology.

6. Entity Translation Scenario. Considering the usage of multilingual ontologies, the revision of the linguistic layer is important for having a high quality artifact. For instance, when a term is translated by non-language experts, a common error is that the translated term is correct from a language point of view (dictionary-based translation), but it is not the optimum one for the domain described by the ontology. 


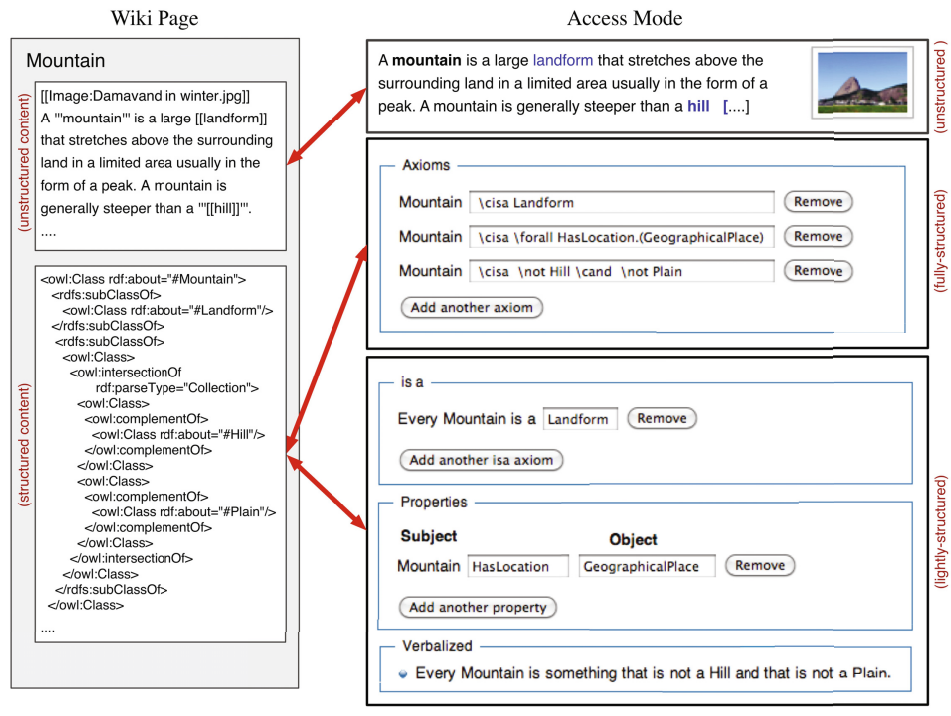

Fig. 1. A page and the access modes in MoKi

Therefore, in this scenario, domain experts and language experts are asked to complete and revise the translations of each entity defined in the ontology by carefully considering the domain described by the ontology.

\section{Supporting the Evolution: The MoKi Tool}

MoK1 is a collaborative MediaWiki-based [4] tool for modeling ontological and procedural knowledge in an integrated manner2. MoKi is grounded on three main pillars, which we briefly illustrate with the help of Figure 1 .

- each basic entity of the ontology (i.e., concepts, object and datatype properties, and individuals) is associated to a wiki page. For instance, the concept Mountain in Figure 1 is associated to a wiki page which contains its description;

- each wiki page describes an entity by means of both unstructured (e.g., free text, images) and structured (e.g. OWL axioms) content;

- a multi-mode access to the page content is provided to support easy usage by users with different skills and competencies. Figure 1 shows three different access modes, for accessing the unstructured and structured content of the wiki page.

A comprehensive description of MoKi is presented in [5].

In order to meet the specific needs of the Organic.Lingua project, MoKi has been customized with the addition of: (i) multilingual services for the automatic translation of

\footnotetext{
${ }^{1}$ http://moki.fbk.eu

2 Though MoKi allows to model both ontological and procedural knowledge, here we will limit our description only to the features for building ontologies.
} 


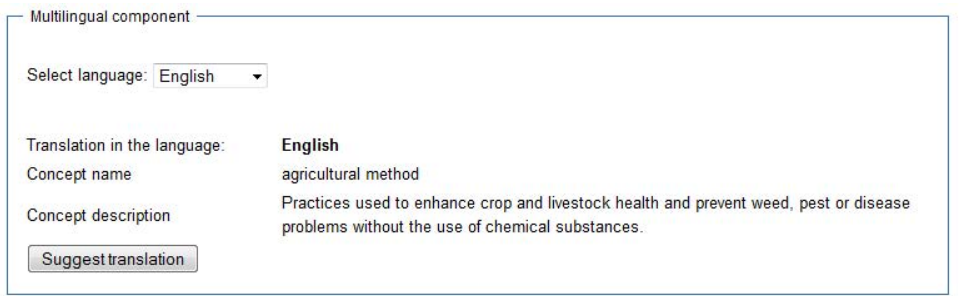

Fig. 2. Multilingual box for facilitating the entity translation

labels and descriptions associated to the ontology entities; and (ii) collaborative features specifically targeting linguistic issues. Translating domain-specific ontologies, in fact, demands that experts discuss and reach an agreement not only with respect to modeling choices, but also to (automated) term translations.

In the context of the Organic.Lingua project, MoKi has been customized with facilities that enable a new type of profile, the Language Expert, to manage the translations carried out on the ontology entities. Such a profile has the role of coordinating the translation activities by approving the terms translation that the other actors involved into the ontology revision process suggest.

The MoKi collaborative nature together with these customizations make it a good technological layer for the application of the multi-role scenario-based methodology.

\subsection{Supporting the Different Scenarios with MoKi}

In this subsection, we briefly describe the main customizations implemented in MoKi with a particular emphasis on how these customizations specifically address the different scenarios described in Section 3

Domain and Language Experts View The semi-structured access mode, dedicated to the Domain and Language Experts, has been equipped with functionalities that permit to accomplish the revisions of the linguistic layer. This set of functionalities permits to revise the translations of names and descriptions of each entity (concepts, individuals, and properties).

For facilitating the browsing and the editing of the translations, a quick view box has been inserted into the mask (as it is shown in Figure 2); this way, language experts are able to navigate through the available translations and, eventually, invoke the third-party translation services for retrieving a suggestion or, alternatively, to edit the translation by themselves (Figure 3).

This customization aims to address all scenarios described in Section 3 .

Approval and Discussion Facilities. Given the complexity of translating domain specific ontologies, translations often need to be checked and agreed upon by a community of experts. This is especially true when ontologies are used to represent terminological standards which need to be carefully discussed and evaluated. To support this collaborative activity we foresee the usage of the wiki-style features of MoKi, expanded with 


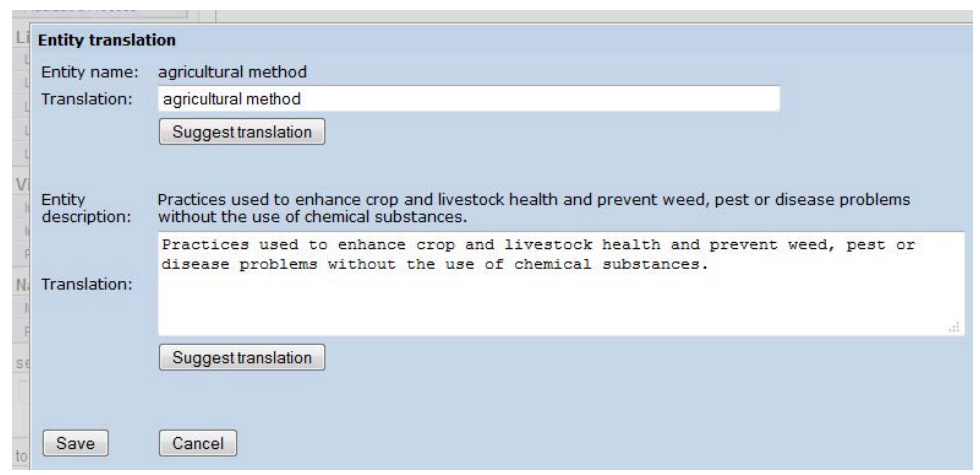

Fig. 3. Quick translation box for editing entities translations

the possibility of assigning specific translations of ontology entities to specific experts who need to monitor, check, and approve the suggested translations. This customization permits to promote the management of the changes carried out on the ontology (in both layers) by providing the facilities necessary to manage the life-cycle of each change.

These facilities may be split in two different sets of features. The first group may be considered as a monitor of the activities performed on each entity page. When changes are committed, approval requests are created. They contain the identification of the expert in charge of approving the change, the date in which the change has been performed, and a natural language description of the change. Moreover, a mechanism for managing the approvals and for maintaining the history of all approval requests for each entity is provided. Instead, the second set contains the facilities for managing the discussions associated with each entity page. A user interface for creating the discussions has been implemented together with a notification procedure that alerts users when new topics/replies, related to the discussions that they are following, have been posted.

This customization aims to address all scenarios described in Section 3 .

Quick Translation Feature. For facilitating the work of language experts, we have implemented the possibility of comparing side-by-side two lists of translations. This way, the language expert in charge of revising the translations, avoiding to navigate among the entity pages, is able to speed-up the revision process.

Figure 4 shows such a view, by presenting the list of concepts in the English and Italian translations. At the right of each element of the table, it is placed a link allowing to invoke a quick translation box (as shown in Figure 3) that gives the opportunity to quickly modify information without opening the entity page. Finally, in the last column, it is placed a flag indicating that changes have been performed on that concept, and a revision/approval is requested.

This customization aims to address the Entity Translation Scenario described in Section 3

Ontology Translator Component. This component manages the translation operations required by MoKi. When a translation, for an entity name or description, is requested, the Ontology Translator invokes the external translation services for performing the 


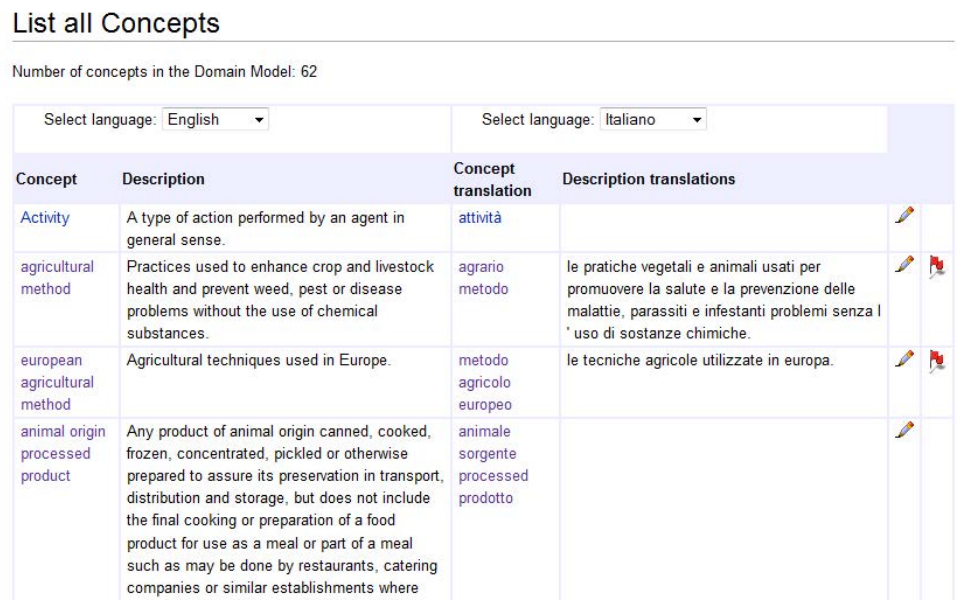

Fig. 4. View for comparing entities translations

translation. The component sends the request to the interface exposed by the third-party translation services and, after the retrieval of the result, the representation of the entity is updated with the information coming from the translation services. Further details, about the translation services used by MoKi can be find in [6].

This customization aims also to address the Entity Translation Scenario.

Interface and Ontology Multilingual Facilities. In order to complete the set of features available for managing the multilingual aspects of the Organic.Lingua project, MoKi has been equipped with two further components that permit to switch between the languages available for the tool interface, to add a new language to the ontology, and to select the language used for showing the ontology in the different views.

Through these facilities, it is also possible to add a new language to the MoKi interface and to manage the translation of its labels. This module has been implemented on top of the multilingual features of MediaWiki.

Instead, concerning the ontology, when a new language is added to the ontology, the Ontology Translator component described above, is invoked for retrieving, for each entity described in the ontology, the translations related to its labels and descriptions.

Finally, the Ontology Export functionality has been revisited by adding the possibility to choose the export languages, among the available ones.

This customization has not been implemented for addressing a particular scenario, but for improving the usability of the tool in a multilingual context.

Linked Open Data Service. In order to permit the exposure of the ontology artifact to the other components deployed on the Organic.Lingua platform, MoKi has been equipped with a service that exposes entity information by using the Linked Open Data format. Such a service permits to perform operations on the ontology remotely; examples of available remote operations are the retrieval of the entire ontology, or of part of it, or the possibility to edit the ontology e.g., by adding a new translated label. The service 
provides a RESTful interface for receiving the requests, while the results are exposed by using the SKOS language 3 .

This customization has not been implemented for addressing a particular scenario, but for linking the tool with the other components deployed on the Organic.Lingua platform.

\section{The Evaluation}

Our goal is evaluating the usage and the usefulness of the MRSB methodology (guiding step by step users through tasks and scenarios) and of the underlying tool, i.e. MoKi, to support different experts in the collaborative evolution of a multilingual ontology. Evolving a multilingual ontology, indeed, adds to the traditional difficulties characterizing the evolution of an ontology, such as the involvement of domain experts (DEs) and their collaboration with knowledge engineers (KEs), also the issues related to the multilinguality, including the need of a third role, the language experts (LEs), and their collaboration with DEs and KEs. In detail, we are interested in answering two main research questions:

RQ1. Is it useful guiding step by step through tasks and scenarios the different experts involved in the collaborative evolution of a multilingual ontology?

RQ2. Do the MoKi functionalities provide an effective support to the the collaborative evolution of a multilingual ontology?

In order to answer these questions we performed two types of analysis: a quantitative and a qualitative one. In the former, data about the activities carried out by the three categories of experts in the context of the evolution of the Organic Agriculture ontology have been analyzed; in the latter, instead, experts have been asked to answer questions aiming at investigating their perception about the usefulness of the MRSB methodology as well as of the MoKi tool in supporting the realization of the different tasks and scenarios foreseen by the MRSB methodology.

Design, Material and Procedure Eleven experts with average experience in their field ranging from 5 to 10 years, were overall involved in the ontology evolution: 3 ontology experts, 4 domain experts and 4 language experts, although one ontology expert and one domain expert also played the role of language experts. The first languages of the 6 LEs were different one from another, thus allowing us to translate the evolved ontology into 6 different languages: Estonian, Spanish, French, Greek, Turkish and Italian.

Most of the experts had no previous knowledge of the tool, hence an initial phase of training was necessary. The training was organized according to the following steps:

- A one-day overall introduction to the tool.

- A few short, on-line, training sessions with the MoKi tool guided by ontology and tool experts, targeted to help domain experts to better understand the capabilities of the tool.

\footnotetext{
3 http: / / www .w3 .org/2004/02/skos /
} 
Table 1. Usage of MoKi by the team of experts for accomplishing the multilingual evolution task

\begin{tabular}{|l|c|c|c|c|c|c|}
\hline $\begin{array}{l}\text { Expert } \\
\text { Category }\end{array}$ & $\begin{array}{c}\text { Entity } \\
\text { Creation }\end{array}$ & $\begin{array}{c}\text { Entity } \\
\text { Update }\end{array}$ & $\begin{array}{c}\text { Entity } \\
\text { Deletion }\end{array}$ & $\begin{array}{c}\text { Entity } \\
\text { Translation }\end{array}$ & $\begin{array}{c}\text { Discussion } \\
\text { Creation }\end{array}$ & $\begin{array}{c}\text { Discussion } \\
\text { Update }\end{array}$ \\
\hline DEs & 52 & 367 & 15 & & 27 & 75 \\
\hline KES & 1 & 50 & 4 & & 3 & 11 \\
\hline LES & & & & 629 & 2 & 24 \\
\hline total & 53 & 417 & 19 & 629 & 32 & 110 \\
\hline
\end{tabular}

- Hands-on usage of the tool: domain experts were left to "play" with MoKi in order to become familiar with the functionalities that they would use during the revision process. This exercise also had the secondary objective to collect doubts and problems encountered by experts.

After the initial training, according to the MRSB methodology, the experts were provided with detailed guidelines (including the description of tasks and scenarios) for the multilingual evolution of the Organic Agriculture ontology. At the end of their ontology evolution activity, experts were asked to fill a questionnaire aiming at investigating their perception about the methodology and the MoKi tool (ease of use, usefulness and capability to support the different scenarios and tasks required by MRSB). Questions were organized in four main parts: (i) one collecting information on the experts' background; (ii) one about the support provided by the methodology used for guiding them in the ontology evolution; (iii) a third one on the subjects' evaluation about MoKi and the role of its different functionalities for accomplishing the MRSB tasks; and (iv) a last one for retrieving information, impressions and questions related to the work performed for the ontology evolution. Some of the questions were provided in the form of open questions, while most of them were closed questions. The latter type mainly concern the experts' evaluation of the tool usefulness on a scale from 1 to 5, varying according to the target of the evaluation (e.g., $1=$ extremely ease/useful/effective, ..., $5=$ extremely useless/difficult/ineffective).

\subsection{Quantitative Evaluation Results}

We analyzed the data on the usage of MoKi during the phases of the project devoted to the evolution of the Organic Agriculture ontology (June - November 2012). Overall each expert spent on average between 8 and 15 hours for accomplishing her work, with peaks of more than 15 hours for DEs. The data are obtained by combining the information stored in the MoKi database and the tool logs. Table 1, reporting the number of the main operations carried out by the team of experts during the ontology evolution phase, shows that the tool has actually been used by all the experts' categories. As reasonable, DEs and KEs have been more involved in the entity editing (creation, update and deletion) and in the discussions (both creation and update), while the LEs have actively participated in the translation activity.

Looking more in detail at the MoKi functionalities exercised by KEs, DEs and LEs, such a trend is overall confirmed (Table 2a). The highest percentage of operations related to the editing and the discussion functionalities has been carried out by DEs, 
while LEs actively exercised the multilingual ones. Surprisingly, the browsing and visualization functionalities have been mainly used by LEs, probably feeling the need to translate labels and descriptions after getting a better understanding of their semantics (through browsing and view functionalities). This result confirms our intuition about the importance of allowing all the different categories of experts involved in the collaborative evolution to easily access to and work on the ontology.

Table 2. Usage of the MoKi functionalities per category of experts and topics of discussions

(a)

\begin{tabular}{|l|l|l|l|}
\hline MoKi Functionality Group & DEs & KEs & LES \\
\hline Discussion and Approval & $46.4 \%$ & $21.6 \%$ & $31.4 \%$ \\
\hline Browsing & $39.2 \%$ & $15.3 \%$ & $45.5 \%$ \\
\hline Multilingual & $33.7 \%$ & $3.4 \%$ & $64.2 \%$ \\
\hline Editing & $61.4 \%$ & $7.8 \%$ & $30.8 \%$ \\
\hline Visualization & $42.7 \%$ & $29.7 \%$ & $45.8 \%$ \\
\hline
\end{tabular}

(b)

\begin{tabular}{|l|c|}
\hline Topic & Percentage \\
\hline Specialization & $35.5 \%$ \\
\hline Mapping to external KBs & $22.6 \%$ \\
\hline Entity Deprecation & $19.4 \%$ \\
\hline Ontology Enrichment & $16.1 \%$ \\
\hline Entity Moving & $3.2 \%$ \\
\hline Definition Rephrasing & $3.2 \%$ \\
\hline
\end{tabular}

Furthermore, inspecting the topics of the discussions allowed us to get a coarse idea of the methodology usage and effectiveness. Table $2 \mathrm{~b}$ reports the percentage of discussions carried out by experts, classified according to the topic. The table suggests that the highest percentage relates to scenarios proposed in the methodology. In detail, most of the discussions had as topic the need to specialize existing entities (Entity Specialization Scenario), followed by those related to the mapping of the Organic Agriculture ontology to external knowledge bases ((Ontology Mapping Scenario) and finally by the deletion of (deprecated) entities and addition of (new relevant) entities. The analysis of the discussions also revealed the effectiveness of the MRSB methodology: only $15 \%$ of the discussions ( 5 out of 32 ) is still open, i.e., no decision has been made yet.

\subsection{Qualitative Evaluation Results}

To investigate the subjective perception of the 11 experts about the support provided by the methodology and the tool to the multilingual ontology evolution activity, we analyzed the subjective data collected through the questionnaire.

In order to evaluate the statistical significance of the positivity/negativity of the collected results we applied the (one-tailed) Mann-Whitney test [7] verifying the hypothesis that $\tilde{F} \leq 3$, where $\tilde{F}$ represents the median of the evaluations for the factor $F$ and 3 is the intermediate value in the 1 to 5 Likert scale. Moreover, to evaluate whether the results are strongly positive, we also applied the same test for the hypothesis that $\tilde{F} \leq 2$, where 2 is the lower level of positive answer in the 1-5 Likert scale. In this case, a significant outcome would mean that, overall, the obtained results are strongly positive. All the analyses are performed with a level of confidence of $95 \%$ (p-value $<0.05$ ), i.e., there is only $5 \%$ of probability that the results are obtained by chance.

Figure 5 (left) reports the distribution of the experts' evaluations about the usefulness of scenarios and tasks used in the MRSB methodology for guiding the experts in their work: scenarios have been mostly judged as absolutely useful, while $10 \%$ of the experts 
revealed minor doubts about the task-based approach. By applying the Mann-Whitney test we found that the usage of scenarios has, also at statistical level, been judged as absolutely useful, while the usage of tasks has been evaluated as useful (with statistical significance of 95\%). The fact that the usage of tasks was less appreciated by LEs and KEs can partially justify the result: their work indeed, that is mainly driven by DEs actions, demands for a less intensive task guidance.
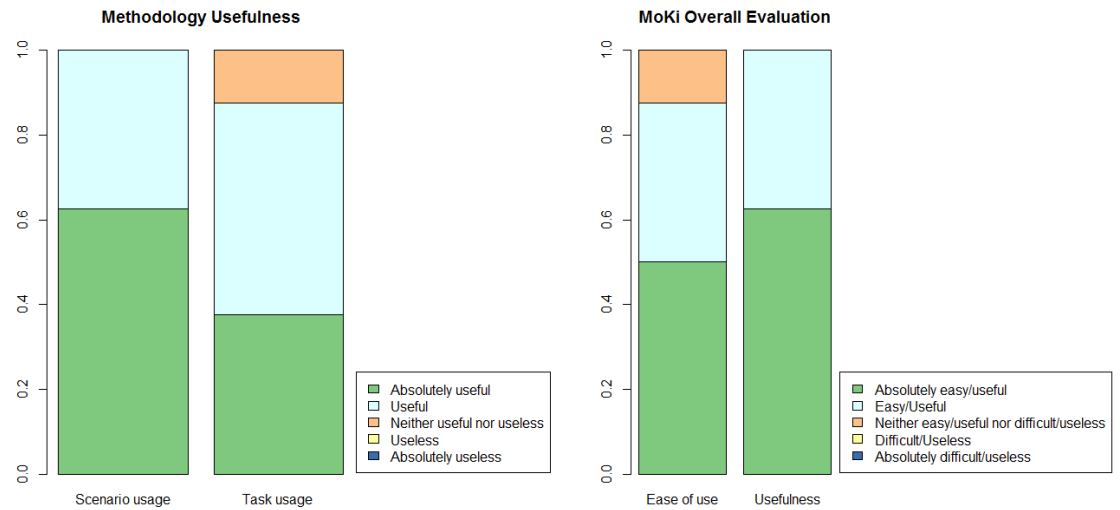

Fig. 5. Experts' evaluation about methodology and MoKi

Figure 5 (right) shows the distribution of the experts' evaluations (on the 5 point scale) of MoKi's ease of use and usefulness for the accomplishment of the ontology evolution activity. Although $10 \%$ of the experts showed doubts about the ease of use of the tool, the evaluations are overall positive (easy) also at statistical level. Moreover, all the experts recognized the usefulness of the tool, resulting in an overall evaluation of MoKi as absolutely useful (also at statistical level). The difficulty in the ease of understanding can be partially explained complementing these results with experts' answers in the open questions: some of the experts asked for a MoKi tutorial. Indeed, while most of the experts have been using MoKi starting from the training phase, a few of them did not immediately practice it, thus finding its usage more difficult later on.

To better understand the relationship between the role of the tool in supporting the methodology used for guiding the experts, we asked the experts to express their evaluation about the effectiveness of the support provided by each typology of functionality to each scenario of the methodology. Table 3 reports the corresponding evaluations according to a $95 \%$ statistical significance, e.g., effective means that there is only a $5 \%$ of possibility that the subjects' evaluations are overall equal or higher than effective by chance. Except for the non-convincing support of the browsing functionalities to the Ontology Mapping Scenario, all the MoKi functionalities have been evaluated as overall at least effective in supporting the MRSB methodology for the multilingual ontology evolution. In detail, the table shows that the discussion functionalities have been considered as absolutely effective in supporting the highest number of scenarios. Indeed, many of the evolution scenarios demand for experts' discussions to reach an agreement. The overall absolute effectiveness of the browsing functionalities has also been assessed in 2 
Table 3. MoKi functionality effectiveness in supporting the MRSB methodology

\begin{tabular}{|c|c|c|c|c|c|c|}
\hline $\begin{array}{c}\text { Functionality } \\
\text { typology }\end{array}$ & $\begin{array}{c}\text { Ontology } \\
\text { enrichment }\end{array}$ & $\begin{array}{c}\text { Entity } \\
\text { deprecation }\end{array}$ & $\begin{array}{c}\text { Entity } \\
\text { Specializ }\end{array}$ & $\begin{array}{c}\text { Entity } \\
\text { Generaliz }\end{array}$ & $\begin{array}{c}\text { Ontology } \\
\text { Mapping }\end{array}$ & $\begin{array}{c}\text { Entity } \\
\text { Translation }\end{array}$ \\
\hline $\begin{array}{c}\text { Discussion } \\
\text { and Awareness }\end{array}$ & $\begin{array}{c}\text { Absolutely } \\
\text { effective }\end{array}$ & $\begin{array}{c}\text { Absolutely } \\
\text { effective }\end{array}$ & Effective & Effective & Effective & $\begin{array}{c}\text { Absolutely } \\
\text { effective }\end{array}$ \\
\hline Browsing & $\begin{array}{c}\text { Absolutely } \\
\text { effective }\end{array}$ & $\begin{array}{c}\text { Absolutely } \\
\text { effective }\end{array}$ & Effective & Effective & $\begin{array}{c}\text { Neither effective } \\
\text { nor ineffective }\end{array}$ & Effective \\
\hline Multilingual & Effective & Effective & Effective & Effective & Effective & $\begin{array}{c}\text { Absolutely } \\
\text { effective }\end{array}$ \\
\hline $\begin{array}{c}\text { Editing } \\
\text { Visualization }\end{array}$ & Effective & Effective & Effective & Effective & Effective & Effective \\
\hline
\end{tabular}

out of the 7 scenarios, despite the moderate support for the Ontology Mapping Scenario. Finally, as expected, the multilingual functionalities have been evaluated as absolutely effective for the Entity Translation Scenario.

\subsection{Findings and Lesson Learned}

The quantitative results reported in Subsection 5.1 show that the MRSB methodology has been actually applied for evolving the Organic Agriculture ontology: the experts' discussions, indeed, were mainly guided by scenario-related topics. Combining these results with the preferences expressed by the experts, we can positively answer RQ1: the MRSB methodology, guiding users step-by-step via scenarios and tasks, provides a concrete help to experts in the evolution of a multilingual ontology, although experts prefer scenarios to tasks.

Together with the application of the MRSB methodology, the quantitative results also show the actual usage of the tool and of its functionalities by the three categories of experts. Moreover, their positive evaluation about ease of use and usefulness of the tool, as well as about effectiveness of the different functionalities in supporting the methodology suggest a positive answer also for RQ2: the MoKi functionalities support the experts in the application of the MRSB methodology for the collaborative evolution of multilingual ontologies.

By further inspecting evaluations and subjects' expertise we found that some relations 4 exist between the evaluations provided by subjects on the effectiveness of the MoKi functionalities with respect to specific scenarios and the typology of expertise of the subject. In particular we found that DEs, differently from the other two categories of experts, perceived the browsing functionalities as more effective in supporting the Entity Deprecation Scenario than the other experts. On the contrary, the KEs found more effective the discussion functionalities for the Entity Specialization Scenario. These results are inline with the overall evaluations of the experts on the effectiveness of the functionalities in supporting the MRSB methodology, i.e., across all the scenarios. Indeed each group of functionalities got an effective average evaluation by all the three categories of experts, except for a higher score by DEs for the browsing functionalities and by KEs for the discussion functionalities.

\footnotetext{
${ }^{4}$ We applied the Anova statistical test to investigate whether the provided evaluations are influenced by the role of the subject.
} 
Finally, the answers to the open questions provided us with suggestions about possible improvements of MoKi. In particular, besides the need to improve the quality of the translation suggestions, features for enhancing the formatting of content and discussion pages, for better supporting concept mappings to external ontologies and for helping experts in the decision making process have been suggested by experts.

Hence, we can conclude that combining a tool easy to use and provided with useful functionalities as MoKi, with a methodology guiding experts step-by-step through concrete scenarios seem to be a winning strategy to overcome the complexity of a problem mixing the two dimensions: the one related to the multilinguality and the one related to the collaboration of different experts.

\section{Related Works}

In this Section, we present a brief review of the main ontology management tools applied to support collaborative creation and sharing of ontological knowledge.

Knoodl 5 facilitates community-oriented development of OWL based ontologies and RDF knowledge bases. It also serves as a semantic technology platform, offering a Java service-based interface or a SPARQL-based interface so that communities can build their own semantic applications using their ontologies and knowledge bases.

Protégé [8] is an open source visual ontology editor and knowledge-base framework. Recently, Collaborative Protégé has been released as an extension of the existing Protégé system. It supports collaborative ontology editing as well as annotation of both ontology components and ontology changes. In addition to the common ontology editing operations, it enables annotation of both ontology components and ontology changes. It supports the searching and filtering of user annotations, also known as notes, based on different criteria.

Semantic MediaWiki+ [9], which includes the Halo Extension, is a further extension on Semantic MediaWiki with a focus on enhanced usability for semantic features. Especially, it supports the annotation of whole pages and parts of text, and offers "knowledge gardening" functionalities, that is maintenance scripts at the semantic level, with the aim to detect inconsistent annotations, near-duplicate entries etc.

The tools above support the collaboration between users for the creation and the evolution of ontologies but do not deal with multilingual issues, which have significantly grown in importance during the last years [10]. To testify the importance of multilinguality in the field of ontology engineering a recent example is provided by the Monnet Project 6 that targets the problem of multilingual information access at the semantic level [11]. Its aim, is to define novel models for cross-lingual information access by using semantic web approaches. Concerning tools, the only instrument supporting the management of multilinguality in ontologies is $\mathrm{NeOn}$ [3]. It is a state-of-the-art, open source multi-platform ontology engineering environment, which provides comprehensive support for the ontology engineering life-cycle. The last version of the toolkit is based on the Eclipse platform and provides an extensive set of plug-ins covering a variety of ontology engineering activities. However, this tool does not provide facilities

\footnotetext{
5 http://www.knoodl.com

${ }^{6}$ http://www.monnet-project.eu
} 
for supporting the multi-role collaboration. Thus, as far as we know, MoKi provides the first significant effort to produce a tool that supports the collaborative evolution of multilingual ontologies, by combining features for the support of collaboration and features for the support of multilinguality and translation.

\section{Conclusions}

In this paper we have presented our experience in applying a scenario-based methodology for modeling complex ontological artifacts composed of a knowledge layer representing the domain, and a linguistic layer making the knowledge available from a multilingual point of view. Such a methodology has been concretely used in the context of the Organic.Lingua EU project with the support of a customized version of the MoKi tool. Three different profiles (domain experts, language experts, and knowledge engineers) evolved the ontology in a collaborative way. Their work together with their subjective evaluation revealed that the synergistic use of the tool and of the methodology permits to evolve the ontology effectively.

\section{References}

1. Stojanovic, L.: Methods and Tools for Ontology Evolution. PhD thesis, University of Karlshrue, Karlshrue, Germany (2004)

2. Flouris, G., Manakanatas, D., Kondylakis, H., Plexousakis, D., Antoniou, G.: Ontology change: classification and survey. Knowledge Eng. Review 23(2), 117-152 (2008)

3. Espinoza, M., Gómez-Pérez, A., Mena, E.: Enriching an ontology with multilingual information. In: Bechhofer, S., Hauswirth, M., Hoffmann, J., Koubarakis, M. (eds.) ESWC 2008. LNCS, vol. 5021, pp. 333-347. Springer, Heidelberg (2008)

4. Wikimedia Foundation: Mediawiki, http://www.mediawiki.org

5. Ghidini, C., Rospocher, M., Serafini, L.: Conceptual modeling in wikis: a reference architecture and a tool. In: eKNOW 2012, Valencia, Spain, pp. 128-135 (2012)

6. Dragoni, M., Ghidini, C., Stoitsis, G., Sicilia, M.A., Sanchez-Alonso, S.: Recommendations for revising existing ontologies and schemas. Deliverable D3.1.1 (2011)

7. Wohlin, C., Runeson, P., Höst, M., Ohlsson, M.C., Regnell, B., Wesslén, A.: Experimentation in software engineering: an introduction. Kluwer Academic Publishers (2000)

8. Gennari, J., Musen, M., Fergerson, R., Grosso, W., Crubézy, M., Eriksson, H., Noy, N., Tu, S.: The evolution of protégé: an environment for knowledge-based systems development. Int. J. Hum.-Comput. Stud. 58(1), 89-123 (2003)

9. Hansch, D., Schnurr, H.P.: Practical applications of semantic mediawiki in commercial environments - case study: semantic-based project management. In: 3rd European Semantic Technology Conference, ESTC 2009 (2009)

10. Peters, C., Braschler, M., Nunzio, G.D., Ferro, N., Gonzalo, J., Sanderson, M.: From research to application in multilingual information access: the contribution of evaluation. In: LREC. European Language Resources Association (2008)

11. McCrae, J., Spohr, D., Cimiano, P.: Linking lexical resources and ontologies on the semantic web with lemon. In: Antoniou, G., Grobelnik, M., Simperl, E., Parsia, B., Plexousakis, D., De Leenheer, P., Pan, J. (eds.) ESWC 2011, Part I. LNCS, vol. 6643, pp. 245-259. Springer, Heidelberg (2011) 